\title{
A novel excitation method for pyroshock simulation
}

\author{
Behnam Houshmand', Alexander Lacher ${ }^{2}$, Nikolas Juengel ${ }^{3}$, \\ Lukas Prasol ${ }^{4}$, Utz von Wagner ${ }^{3}$ and Eckart Uhlmann ${ }^{4}$
}

\begin{abstract}
Pyroshocks are structural responses to transient excitation caused by the essential use of pyrotechnic devices in aerospace applications. In order to avoid damage in aerospace structures due to pyroshocks, tests are performed on earth prior to launching space modules. In these tests, explosive loads are often replaced by alternative excitation methods such as hammer pendulums or shakers simulating on earth the impact taking place in space. However, there does not yet exist an adequate excitation method satisfying all requirements of a fast, reliable, predictable and repeatable test setup. Whereas hammers are poorely controllable in terms of generating desired shock spectra, shakers show limitations in terms of the bandwidths of up to $10 \mathrm{kHz}$ which are prescribed in the test specifications.

The authors present a novel contactless and non-destructive excitation method for pyroshock test devices based on a mechatronic coupling by applying Lorentz forces to the carrying structure. For generating the corresponding magnetic field, the capacitor of a Resistor-Inductor-Capacitor RLC resonator circuit is initially charged and then discharged leading to high currents in the coil which is placed close to the carrying structure. Latter is then inducing a counter current in the aluminum structure which reacts with high multidirectional Lorentz forces. Any adjustments are done by tuning the properties of the circuit such as initial charge, capacitance and inductance. By connecting several different coils, frequency modulation and by splitting the currents more complex signals can be generated matching the natural frequencies of the structure. Almost all disadvantages of common excitation methods are eliminated by the proposed mechanism.
\end{abstract}

\section{Keywords}

Pyroshock, simulation, excitation, wave propagation, SRS, magnetic field, Lorentz force, RLC resonator

\section{Introduction}

Common existing pyroshock test facilities are described in various references such as Bernaudin et al. (2008), Davie and Bateman (1995), Henderson and Piersol (2003) and Lalanne (2005). The most recent developments are found in Bäger (2009), Dwyer and Moul (1988), Filippi et al. (1999), Kiryenko et al. (2005), Schweickert (1997) and Smith (1986) which all have the goal of simulating far and mid field pyroshocks in order to meet the strict requirements of the pyroshock test specifications in terms of the shock response spectrum (SRS), see e.g. Bernaudin et al. (2008). An overview of further articles concerning pyroshock themes can be found in Lee et al. (2012). Far and mid field pyroshocks address signal bandwidths of up to $10 \mathrm{kHz}$ whereas all devices presented are based on a mechanical impact between a striker and a structure carrying the test specimen which is either performed in-plane or out-of-plane. The approximate maximum acceleration levels reach up to $5000 \mathrm{~g}$ resulting in very high amounts of energy which have to be transferred to the structure within very small time periods. In the

\footnotetext{
'AneCom AeroTest GmbH, Design and Analysis Department, Wildau, Germany

${ }^{2}$ Rolls-Royce Deutschland, Rotatives Department, Blankenfelde-Mahlow, Germany

${ }^{3}$ Department of Applied Mechanics, Technische Universität Berlin, Germany

${ }^{4}$ Institute for Machine Tools and Factory Management, Technische Universität Berlin, Germany

Received: 25 March 2014; accepted: 19 November 2014

Corresponding author:

Alexander Lacher, Rolls-Royce Deutschland, Rotatives Department, Blankenfelde-Mahlow, Germany.

Email: alexander.lacher@rolls-royce.com
} 
existing test, setups based on mechanical impact are categorized into four different groups which are (a) sphere against rod, (b) sphere against plate, (c) sphere against disk and (d) rod against disk. Cases (a) and (c) have been investigated by developing a semi-analytical solution procedure, see Lacher et al. (2012a), whereas computation algorithms of cases (b) and (d) can be found in Lacher et al. (2011) and Lacher (2011). Additionally, a recently published detailed analytical study of different impact scenarios based on a CPU time saving asymptotic approach can be found in Caresta et al. (2014). The corresponding algorithms allow for a rough prediction of accelerations and resulting SRS on carrying structures at arbitrary points in terms of the impact by a striker. FE based investigations of pyroshock tests can be found in e.g. Barboni et al. (2003), Lacher (2012b) and Kiryenko et al. (2005).

Next to the mentioned passive mechanical strikers, also electrodynamic shakers and (based on investigations conducted at Chair of Mechatronics and Machine Dynamics at TU Berlin) piezoelectric staple actuators can be used in order to transfer shocks into carrying structures, see Houshmand (2010) and Roggan (2012). In both methods, signals can be designed by using input voltages in terms of wavelets, see e.g. Bernaudin et al. (2008). In some applications, explosives are used in order to achieve high acceleration levels. The range of presently existing excitation methods for pyroshock simulation can thus be written as (1) explosives, (2) mechanical strikers, (3) electrodynamic shakers and (4) piezoelectric staple actuators. In Jang and Lee (2014) a laser-induced shock excitation gives first promising results in terms of a new non-destructive excitation method but has to be further expanded for test application purposes. Also, in Stewart et al. (2014) a hydraulic blast simulator is presented as an alternative way to common explosives. However, the degree of destruction of the involved materials is non negligible.

Whereas case (1) is delimited by the controllability and repeatability of the test procedure, case (3) shows its limitations at frequencies higher than $3 \mathrm{kHz}$ where shakers tend to demand extremely high power. Although as well controlable as shakers, piezoactuators (4) are limited with respect to the acceleration amplitude in the SRS graph. So far, no excitation method other than a mechanical striker (2) is able to meet the common requirements of reliable test setups for reaching the high acceleration levels at high frequency bandwidths up to $10 \mathrm{kHz}$. However, its main disadvantages are the poor controllability caused by its laborious handling and the fact that influencing the shock signal is related to change hammer head materials, radius of curvature impact velocity and so on.

Therefore, the authors present a novel contactless excitation method combining advantages of all methods mentioned before. Its working principle is inspired by high speed forming technology, see Uhlmann and Ziefle (2010), Unger et al. (2006) and $\mathrm{Xu}$ et al. (2008), based on a simple $R L C$ resonator circuit charged at a specific voltage. During the discharging process, the resonator's coil transfers a transient magnetic pressure to a carrying metal plate leading to a Lorentz volume force. This very high dynamic load results in the propagation of transversal and longitudinal waves in the carrying structure and the test specimen. First tests revealed that, as an example, SRS peak levels of up to $5000 \mathrm{~g}$ are obtained on a $80 \mathrm{~kg}$ carrying plate by charging a capacitor bank by an energy amount of $1 \mathrm{~kJ}$. In fact, the plate stayed entirely unaffected and no evidence of plastic deformation in terms of indentation or damage could be found afterwards. In addition, the magnetic field produced during the electrical impact is, due to its strong spatial decaying characteristics (order of $\mathrm{r}^{-3}$ in terms of the distance $r$ from the coil), not assumed to affect any electrical components of the test specimen.

Further refinements of the technology are investigated such as connecting higher numbers of $R L C$ resonator circuits additionally switched by field-effect transistors in order to produce wavelets.

\section{Experimental setup and working principle}

In order to achieve high acceleration levels on the carrying plate, the experimental setup (see Figure 1) requires a power electronic pulse generator (here: FA1440-60-SW from Chair of Machine Tools and Factory Management, TU Berlin, see Figure 2) based on an $R L C$. The pulse-like excitation of the aluminum plate (placed on non-locating bearings) is realized by a flat axis-symmetric coil, see Figure 2. The coil is located outside the device on a rigid foundation beneath the aluminium plate (Figures 1 and 3(a)). In a first step, the capacitor banks are charged by an arbitrary

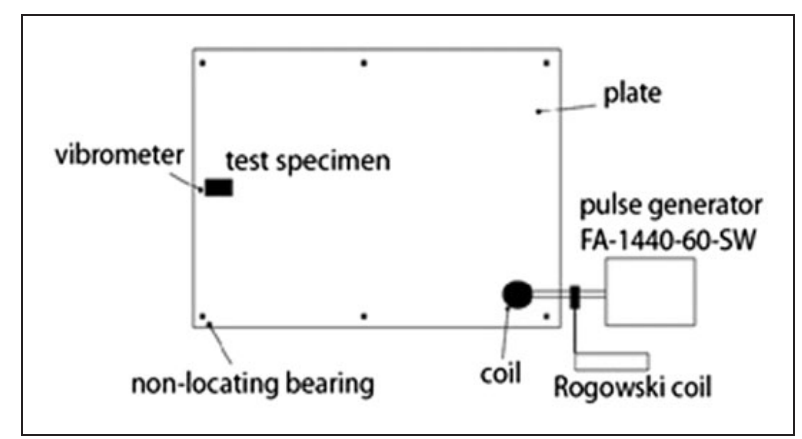

Figure I. Setup of the proposed pyroshock test facility (top view). 


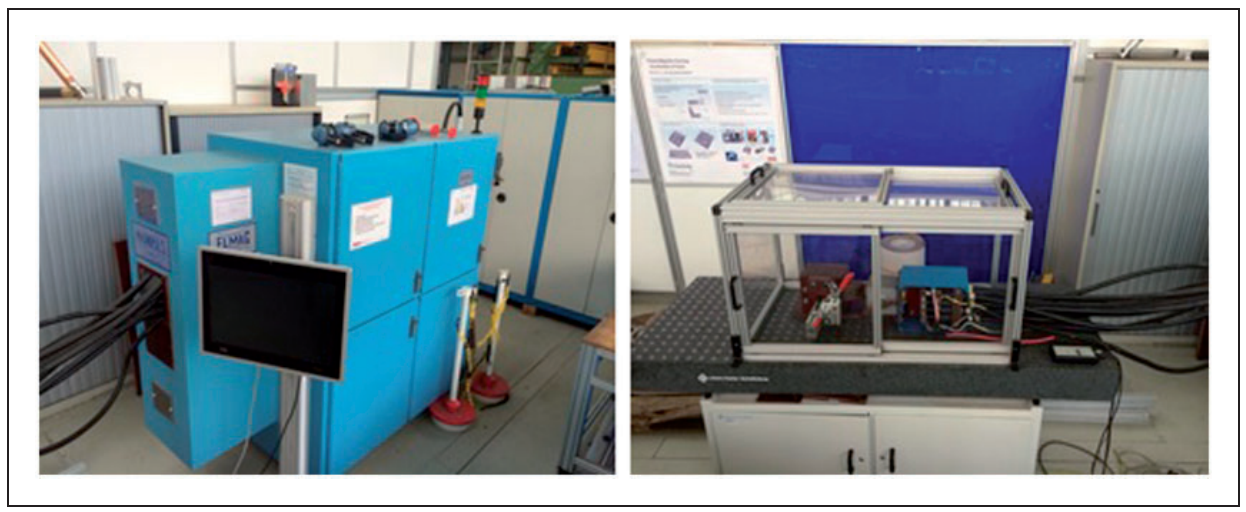

Figure 2. Instrumentation utilized, pulse generator (left) and coil (right). Reproduced with kind permission from the Institute for Machine Tools and Factory Management (IWF), TU-Berlin.

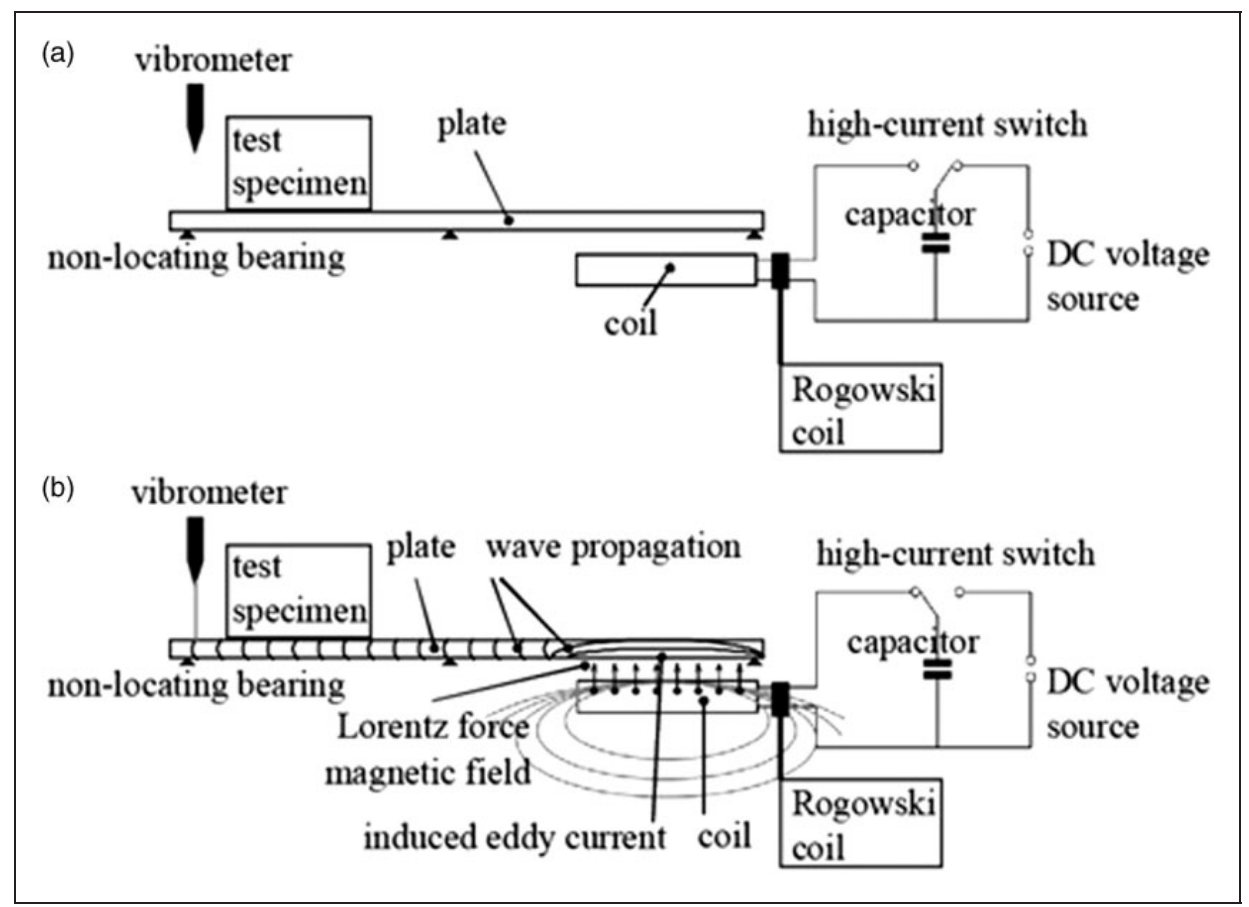

Figure 3. Working principle of the proposed pyroshock test facility (side view), (a) charging and (b) discharging process.

amount of energy (which can be used to control the force amplitude), see Figure 3(a). By the use of a high-current switch, the capacitors are discharged leading to large currents (up to several hundred $\mathrm{kA}$ ) that generate an intense magnetic field inside the tool coil (Figure 3(b)).

This magnetic field induces eddy currents at the surface of the plate which are running in the opposite direction compared to the primary currents in the tool coil. Due to the current, inside the coil a Lorentz volume force distribution is generated acting on the aluminum plate. A Rogowski coil and a vibrometer are used for process measurement. The Rogowski coil measures induced sinusiodal currents during the discharging process whereas the vibrometer detects the velocity at a point on the carrying plate (or specimen) occurring during the excitation process. Because of the modular arrangement of the pulse generator's capacitors, it is possible to vary the system's capacity. Hence, beside the amplitude also the discharging frequency of the excitation process can be controlled.

\section{Magneto - thermo - structural modeling}

Developing a multifield model making pyroshock test results predictable according to the proposed method 
requires the complex interaction of multiple fields in physics. In the presented excitation technique the coil of a discharge circuit is exciting an aluminium carrying plate by repulsive transient Lorentz volume forces which are produced by the discharged current. The Lorentz volume forces result in the propagation of mechanical waves through the carrying structure leading to an acceleration field. In terms of the purpose of pyroshock testing, the setup can be used as a test facility at which the input parameters are basically electrical ones such as the charging voltage and the circuit characteristics, finally leading to a mechanical field output in terms of the SRS. A multifield FE-model has been developed which actively couples the electromagnetic, thermal and structural fields. The model deals with quasi-static Maxwell equations, impulse and energy balances instantly. The discharging process takes about $200 \mu$ s which is slow enough to allow for the assumption of quasi-static electromagnetism. For describing the wave propagation, dynamic momentum balances are considered. The resistive heat generated due to induction propagates in the medium according to Fourier's heat transfer law. Thermal analysis finds its importance in the fact that material softening may

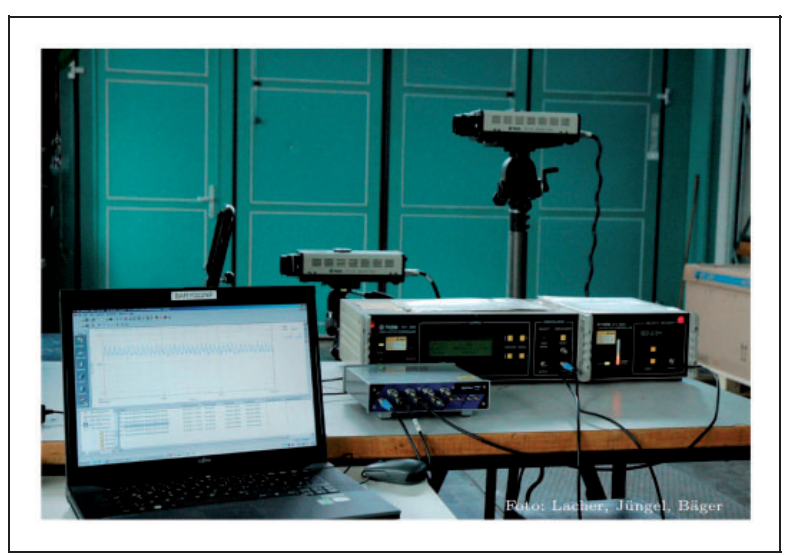

Figure 4. Laser Vibrometers and digital signal processing devices utilized. Reproduced with kind permission from Alexander Lacher (Lacher, 20II). appear locally in the vicinity of the exciting electrodynamic coil if the testing object is large compared to the coil's dimensions. In this case, an enormous discharging energy is required in order to achieve the high acceleration levels of the pyroshock test specifications. As also practiced in metal forming processes which are based on considerably higher energy amounts, the authors decided to include thermal coupling in the model. Lorentz volume forces are strongly dependend on the coil's geometry which influences the inductance and, consequently, the force distribution. As it will be presented in the following section, the discharge frequency depends on the total inductance, capacity and active resistance which requires a study of the discharge circuit.

\section{I. Excitation circuit}

Figure 5 represents the diagram of the excitation circuit. In the primary circuit $C, L_{i}$ and $R_{i}$ denote for the equivalent capacity of the capacitor banks charged by the voltage $U_{0}$, the total inner inductance and the resistance of the high voltage discharge machine respectively. $L_{1}$ and $R_{1}$ represent the self inductance and electrical resistance of the coil. Accordingly, in the secondary circuit $L_{2}$ and $R_{2}$ assume to be the active self inductance and resistance of the carrying plate. $M$ represents the mutual inductance between the coil and the testing object varying strongly with the initial distance and material between coil and carrying plate.

For a better understanding of the discharging process, the excitation circuit (Figure 5) can be replaced by an equivalent $R L C$ resonator circuit, as presented in Figure 6.

With the assumption of both, negligible geometrical and material nonlinearity in all three fields, the $R L C$ resonator circuit can be considered having constant resistive, inductive and capacitive elements, which, as described e.g. in Winkler (1973), has the well known analytical solution for its resulting linear differential equation

$$
\frac{d}{d t}\left(L_{i}+L_{a}\right) I_{1}(t)+\left(R_{i}+R_{a}\right) I_{1}(t)+\int_{0}^{t} \frac{1}{C} I_{1}\left(t^{\prime}\right) \mathrm{d} t^{\prime}=U_{0}
$$

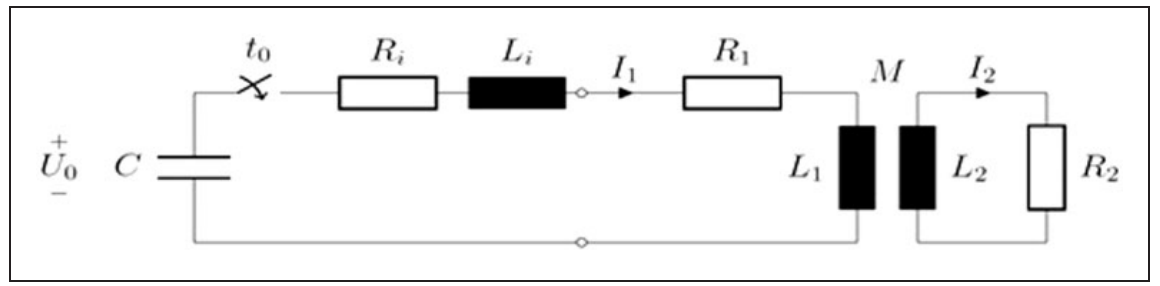

Figure 5. Scheme of excitation circuit. 
with

$$
L_{a}=L_{1}-\left(\frac{M}{L_{2}}\right)^{2} L_{2}, \quad R_{a}=R_{1}+\left(\frac{M}{L_{2}}\right)^{2} R_{2}
$$

The resulting discharge currents for the primary and secondary circuit can be written as follows

$$
\begin{aligned}
& I_{1}(t)=U_{0} \omega C e^{-\delta t} \sin \omega t, \\
& I_{2}(t)=-\frac{M}{L_{2}} U_{0} C\left[\alpha e^{-\alpha t}+\omega e^{-\delta t} \sin (\omega t+\varphi)\right]
\end{aligned}
$$

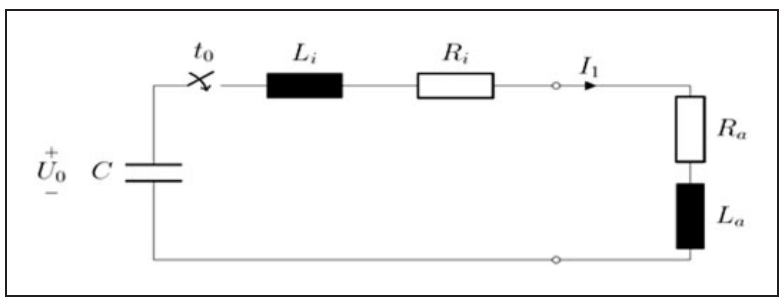

Figure 6. Equivalent of excitation circuit. in which

$$
\begin{aligned}
& \omega=\sqrt{C\left(L_{i}+L_{a}\right)}, \quad \alpha=\frac{R_{2}}{L_{2}}, \quad \delta=\frac{1}{2} \frac{R_{i}+R_{a}}{L_{i}+L_{a}}, \\
& \varphi=\sin ^{-1}\left(\frac{R_{2}}{L_{2}} \sqrt{C\left(L_{i}+L_{a}\right)}\right)
\end{aligned}
$$

\subsection{Numerical model}

Figure 7 schematically shows the numerical simulation procedure.The FE simulation considers the interactive coupling of the magnetic, thermal and structural fields sequently for each timestep which allows a realistic approach of the system's behaviour. In order to carry out such a simulation four physics environments are defined.

The useful outputs of the first electromagnetic analysis are the Joule heat generation rate [Watt] and the Lorentz Forces. In the sequent thermal analysis the heat generation rate of the pervious electromagnetic analysis is interpolated onto the thermal domain in order to calculate the temperature distribution. Sequently, in the structural analysis the Lorentz forces will interpolated from the previous electromagnetic analysis utilizing the temperature distribution from the previous thermal analysis. The primary

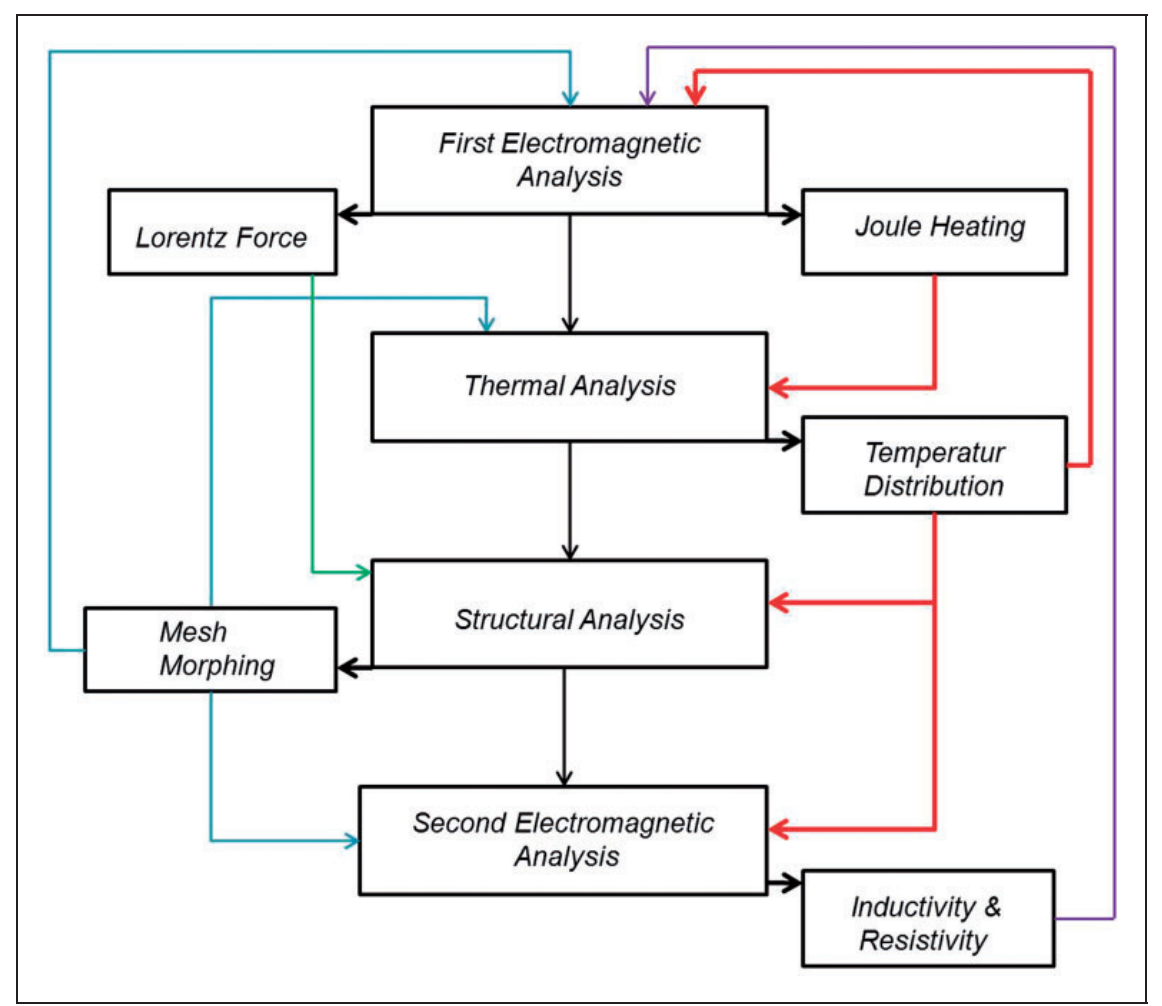

Figure 7. Schematic diagram. 


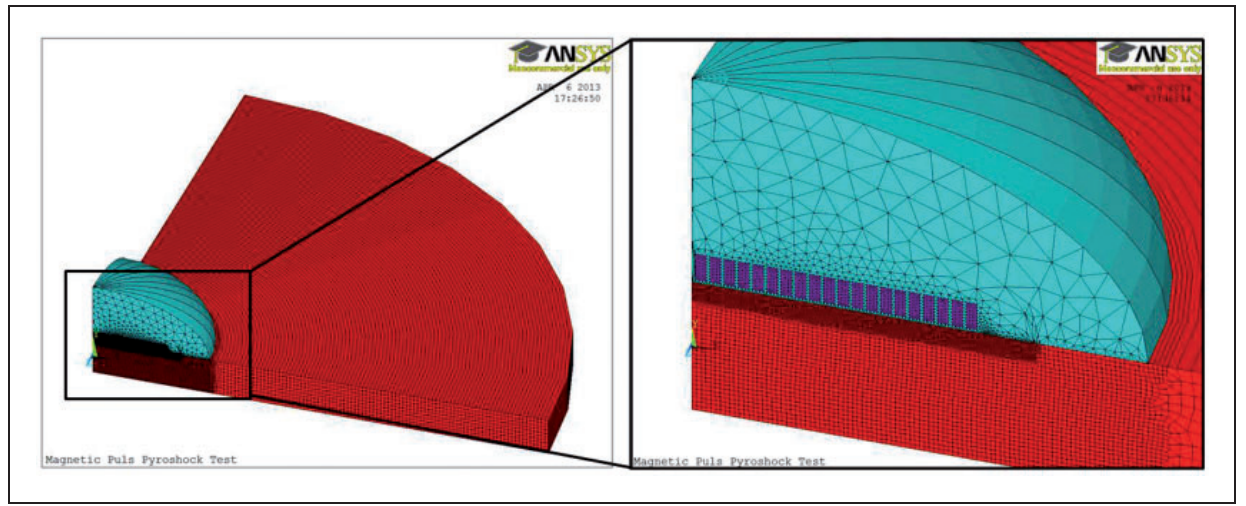

Figure 8. Finite element model.

output of the structural analysis are the displacements of the test specimen and the coil, which may be large enough to effect the inductivities and resistivities. In addition, the resulting themperature distribution from the thermal analysis can also change the resistivities and inductivities. To monitor this effect the second electromagnetic analysis was implemented in each loop in order to calculate the resistivities and the inductivities. The displacements which were calculated in the previous structural analysis are used to update the geometries of both the first and second electromagnetic analysis and also the thermal analysis (this geometry update is called mesh morphing). Having this quick introduction of the simulation loop (see Figure 7), it is important to look into the theoretical background of each physics environment in more detail.

The first physics environment contains a magnetostatic analysis with static domain for air, conductor domain for coil and eddy current domain for plate elements to calculate the Lorentz forces on coil and plate and the heat generation rate due to joule heating.

For a homogeneous, isotropic electroconductive material, like most metals, the Lorentz forces can be described as a cross product of the current density vector $\mathbf{j}(\mathbf{x}, t)$ and the magnetic flux vector $\mathbf{B}(\mathbf{x}, t)$ by means of

$$
\mathbf{f}(\mathbf{x}, t)=\mathbf{j}(\mathbf{x}, t)\left[\frac{A}{\mathrm{~m}^{2}}\right] \times \mathbf{B}(\mathbf{x}, t)\left[\frac{N}{A \cdot \mathrm{m}}\right],
$$

whereas the total excitation force $F(t)$ results from integration over the volume $\mathbf{V}_{2}$ of the carrying plate as follows

$$
F(t)=\int_{\mathbf{V}_{2}} \mathbf{f}(\mathbf{x}, t) \mathrm{d} v
$$

Table I. Material properties.

\begin{tabular}{llll}
\hline Material properties & Air & Copper & Aluminium \\
\hline Relative permeability $[\mu]$ & $\mathrm{I}$ & 0.999 & 1.00002 \\
Electrical resistivity $\rho_{\mathrm{e}}[\mathrm{n} \Omega \mathbf{n m}]$ & - & 17 & 28 \\
Thermal conductivity $\mathrm{k}\left[\mathrm{Wnm} \mathrm{nm}^{-1} \mathrm{n} K\right]$ & - & 400 & 237 \\
Specific heat $C\left[\mathrm{j} \mathrm{ng}^{-1} \mathbf{n} \mathrm{K}^{-1}\right]$ & - & 390 & 910 \\
Mass density $\rho\left[\mathrm{kgm}^{-3}\right]$ & $\mathrm{I}$ & 8900 & 2700 \\
Young's modulus $E[\mathrm{GPa}]$ & - & 115 & 70 \\
Poisson's ratio $v[]$ & - & 0.34 & 0.35 \\
\hline
\end{tabular}

\begin{tabular}{|c|c|c|c|c|c|c|c|}
\hline Setup & $\begin{array}{l}E_{c} \\
[k]]\end{array}$ & $U_{0}[k V]$ & $L_{a}[\mu H]$ & $C[\mu F]$ & $R_{a}[\Omega]$ & $\omega\left[s^{-1}\right]$ & $\delta\left[\Omega \mathbf{n} H^{-1}\right]$ \\
\hline I & 0.4 & 1.825 & 2.183 & 240 & 0.0616 & 45.87 & 14116 \\
\hline 2 & 0.8 & 2.582 & 2.183 & 240 & 0.0616 & 45.87 & 14116 \\
\hline 3 & 1.3 & 3.291 & 2.183 & 240 & 0.0616 & 45.87 & 14116 \\
\hline 4 & 0.8 & 1.825 & 2.063 & 480 & 0.0431 & 33.30 & 10453 \\
\hline 5 & 1.3 & 1.972 & 1.03 & 700 & 0.0185 & 37.70 & 8980 \\
\hline
\end{tabular}

Table 2. Circuit setups for parameter study.

The equation (5) serves as the basic magneto-structural coupling equation between the excitation circuit and the carrying plate.

To calculate the magnetic flux density vector $\mathbf{B}(\mathbf{x}, t)$, the magnetic field intensity vector $\mathbf{H}(\mathbf{x}, t)$, the electric flux density vector $\mathbf{D}(\mathbf{x}, t)$, the electric field intensity vector $\mathbf{E}(\mathbf{x}, t)$ and the electric current density $\mathbf{j}(\mathbf{x}, t)$ in continua with the assumption that the electric flux density vector $\mathbf{D}(\mathbf{x}, t)$ is constant in time, the quasistatic Maxwell's equations

$$
\operatorname{rot} \mathbf{H}(\mathbf{x}, t)=\mathbf{j}(\mathbf{x}, t)
$$




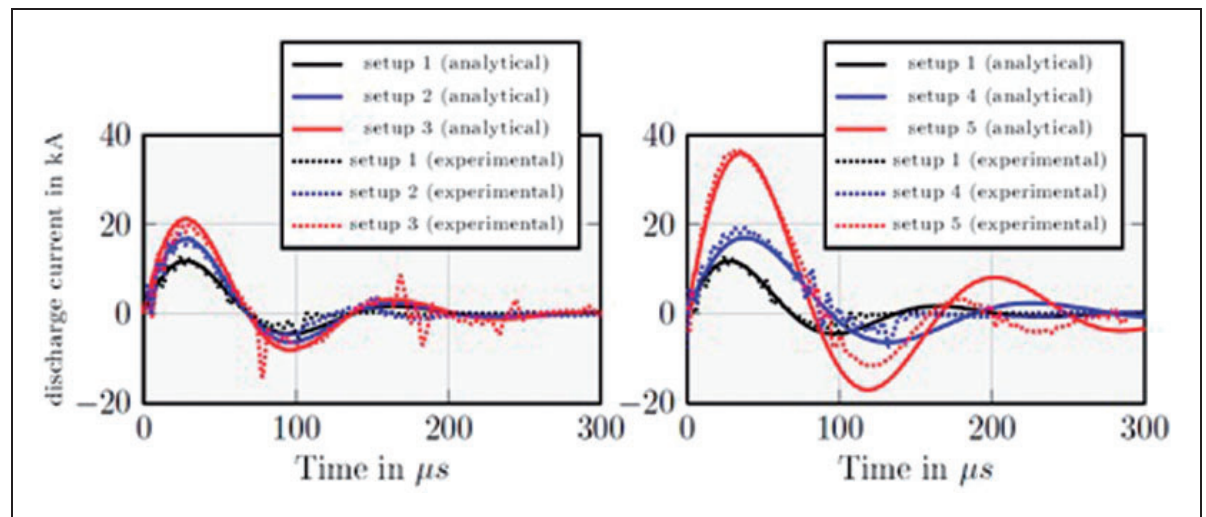

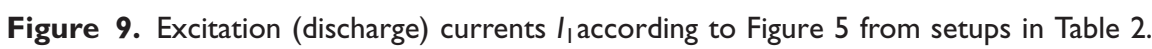

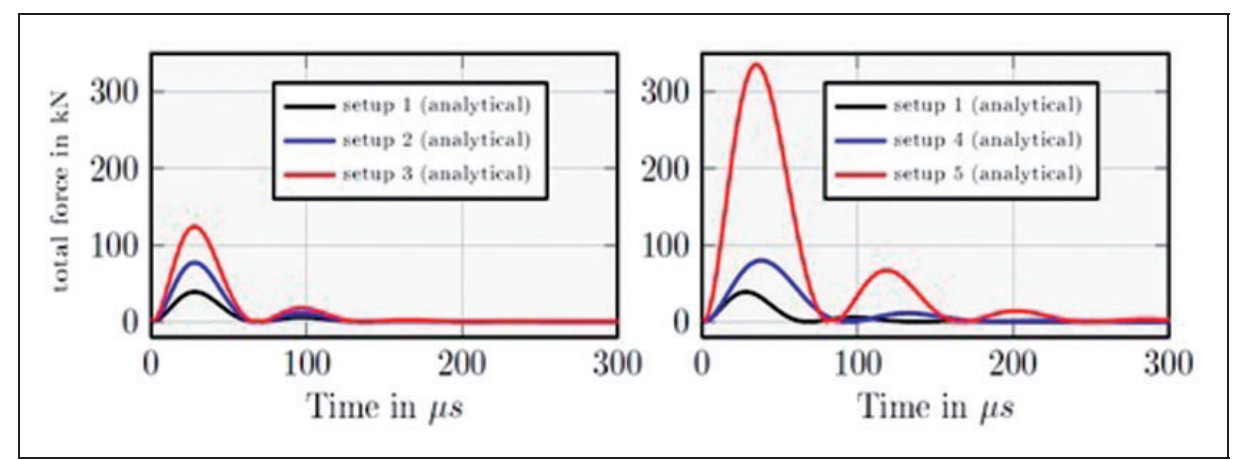

Figure 10. Simulated force $F(t)$ on a circular plate resulting from Lorentz body forces according to (6) from setups in Table 2.

are applicable. In order to study the excitation and the system's response by taking into account the considerations shown in Figure 7, a $2 \mathrm{D}$ rotation-symmetric finite element model was developed, using the Ansys APDL (Ansys Parametric Design Language) software. It is illustrated in Figure 8, in which the hemisphere on top of the model, the circluar section on the bottom and the small rectangulars denote for the air, the aluminium plate and the copper coil's rings respectively (for material properties see Table 1).

In the present simulation of circular coil and plate (diameter $D=1 \mathrm{~m}$, thickness $h=0.04 \mathrm{~m}$ ) the eddy current density has tangential components only whereas the magnetic flux vector is directed in radial and axial direction. Therefore the correlation between the induced eddy current in the carrying plate $\boldsymbol{j}_{2}(\mathbf{x}, t)$ and it's equivalent in excitation circuit from Figure 5 is described as follows

$$
I_{2}(t)=\int_{\mathrm{A}_{2}} \mathbf{j}(\mathbf{x}, t) \cdot d \mathbf{n}(\mathbf{x}, t) \text { and } d \mathbf{n}(\mathbf{x}, t)=d \mathrm{~A}(\mathbf{x}, t) \mathbf{e}_{\Phi}(\mathbf{x}, t)
$$

with $\mathbf{x}=\{r, \Phi, z\}$ representing the position of each material point in cylindrical coordinates and the area $\mathrm{A}_{2}$ representing the cross section area of the test object. According to equation (5) with equation (3) and considering the correlation (equation (8)), the mechanical excitation $\mathbf{f}(\mathbf{x}, t)$ takes place at discharge frequency $\omega$ at a phase difference of $\varphi$ with respect to the discharge current in the coil. The phase difference depends on the resistance $R_{2}$ of the carrying plate varying with its thickness. For a thick testing object the resistance is negligible and, consequently, there is no phase difference between the discharge current in the coil and the excitation, see also Winkler (1973) and $\mathrm{Xu}$ et al. (2008). Additionally, it is important to mention that, concerning the coil's FE modeling, the authors assume each of the 20 concentric copper rings (small rectangulars in r.h.s. of Figure 8) to be one separate coil with one separate winding, separate dimensions and discharging circuit. In order to approach one flat coil in the simulation, all 20 rings are linked to one single current $I_{1}$.

A thermal transient analysis was applied as the second physics environment for coil and plate elements 


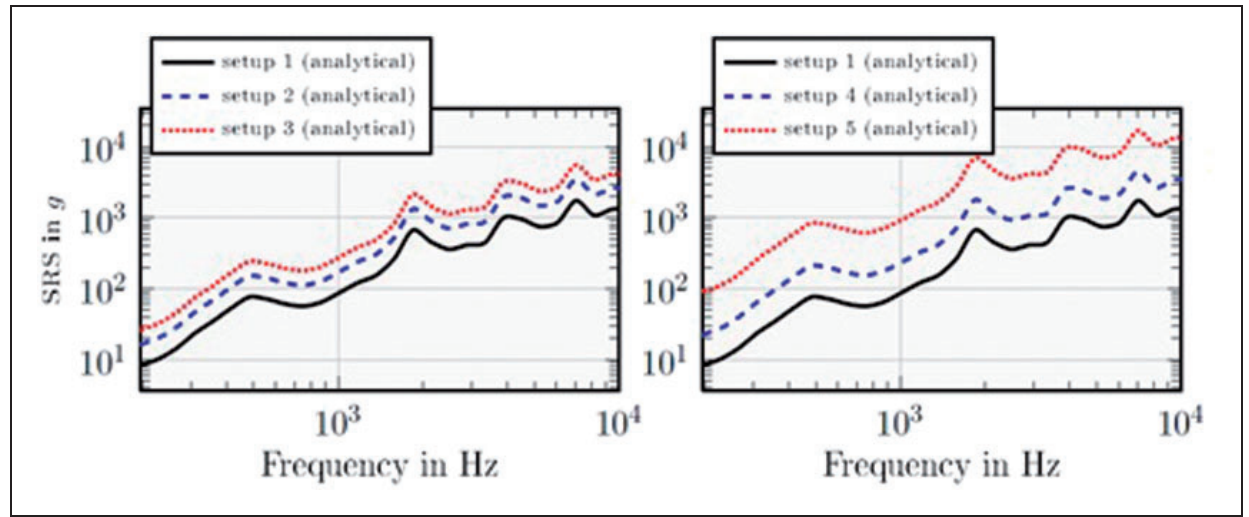

Figure II. Simulated SRS at point P from Figure 8, circular plate.

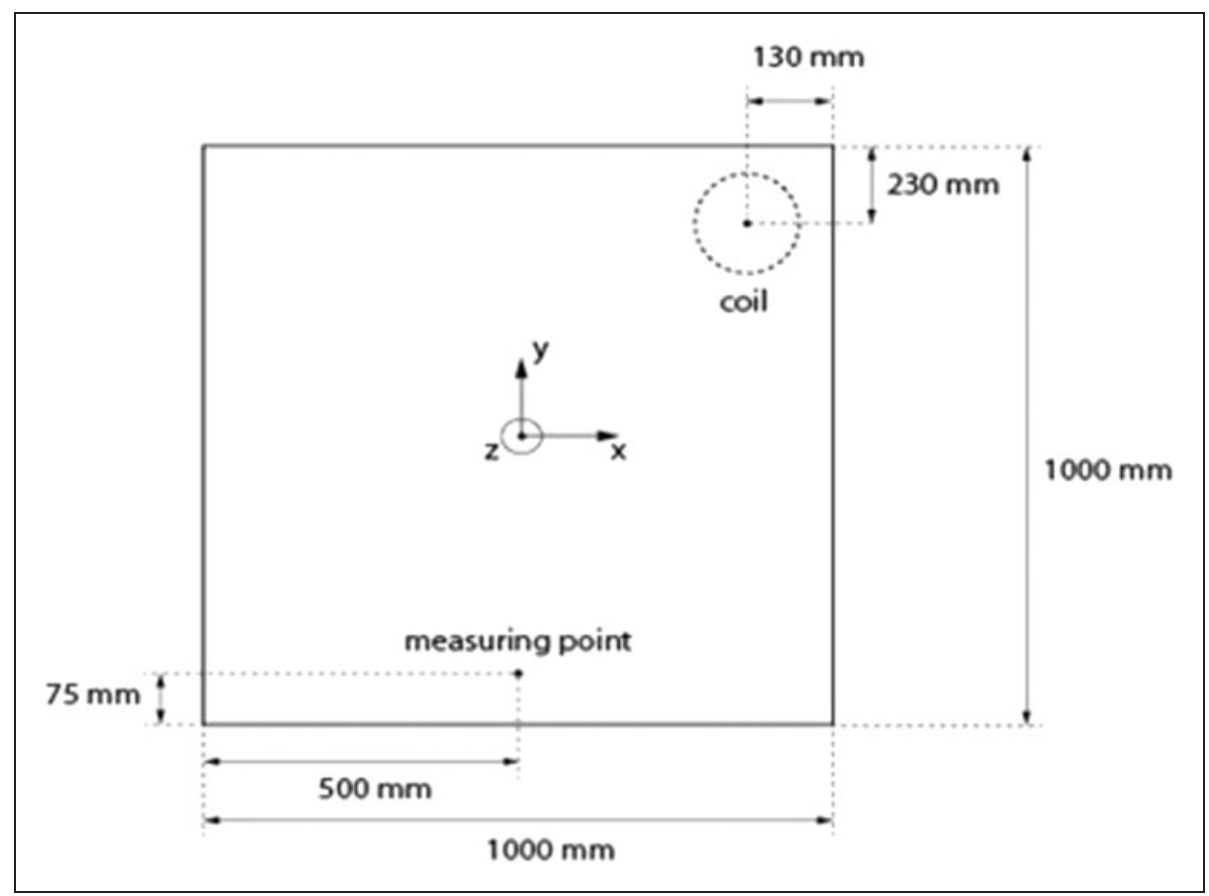

Figure 12. Experimental setup; locations of measuring point and coil (top view).

to calculate the temperature distribution due to Joule heating. Consequently a structural transient analysis as the third physics environment was used to calculate the displacements considering the balance of momentum after discretization

$$
\{M\}\{\ddot{\mathrm{u}}\}+\{D\}\{\dot{\mathrm{u}}\}+\{K\}\{\mathrm{u}\}=\{f\}
$$

in which $\{M\},\{D\}$ and $\{K\}$ are the global mass, damping and stiffness matrixes. Vector $\{u\}$ is the global displacement vector and $\{f\}$ is the descretized Lorenz forces vector. Finally, the last physics environment contains a magnetostatic analysis with static domain for all elements to calculate the active inductance and resistance of the system in each timestep.

At the end of each loop, the nodal coordinates of each node were updated to the resulting displacement to be used in the magnetostatic and thermal analysis. It is important to point out the fact that the nodal coordinates for each node had to be reset to material coordinates before carrying out the structural analysis to prevent the wrong calculation of the stresses. The physical properties of the materials are assumed to be constant due to the very low changes in temperature. On the first sight, this assumption seems to be in contradiction to the discussion in the beginning of this section 


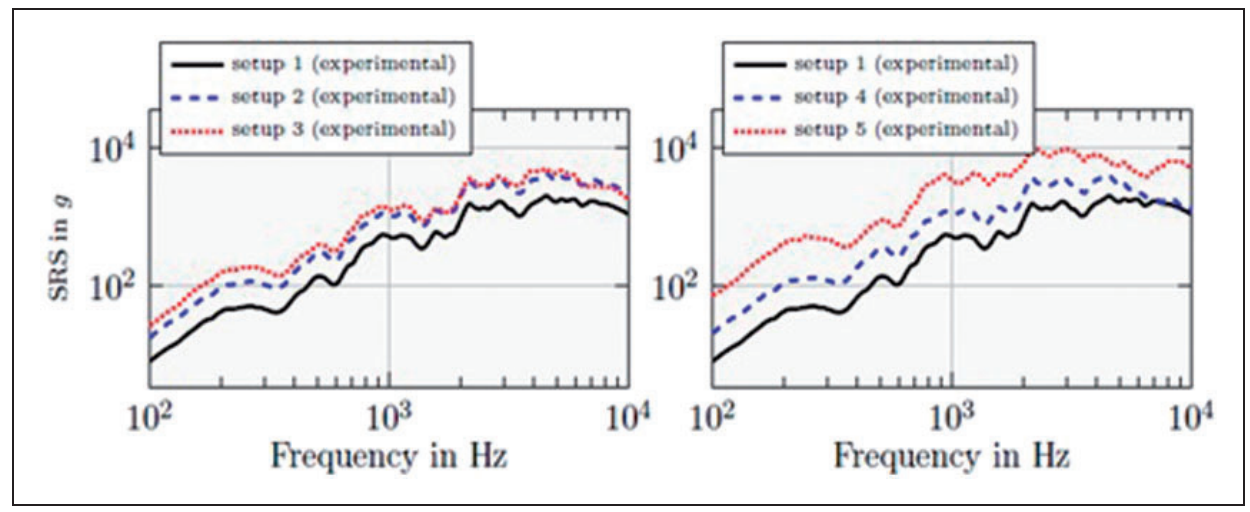

Figure 13. SRS from experiment, rectangular plate.

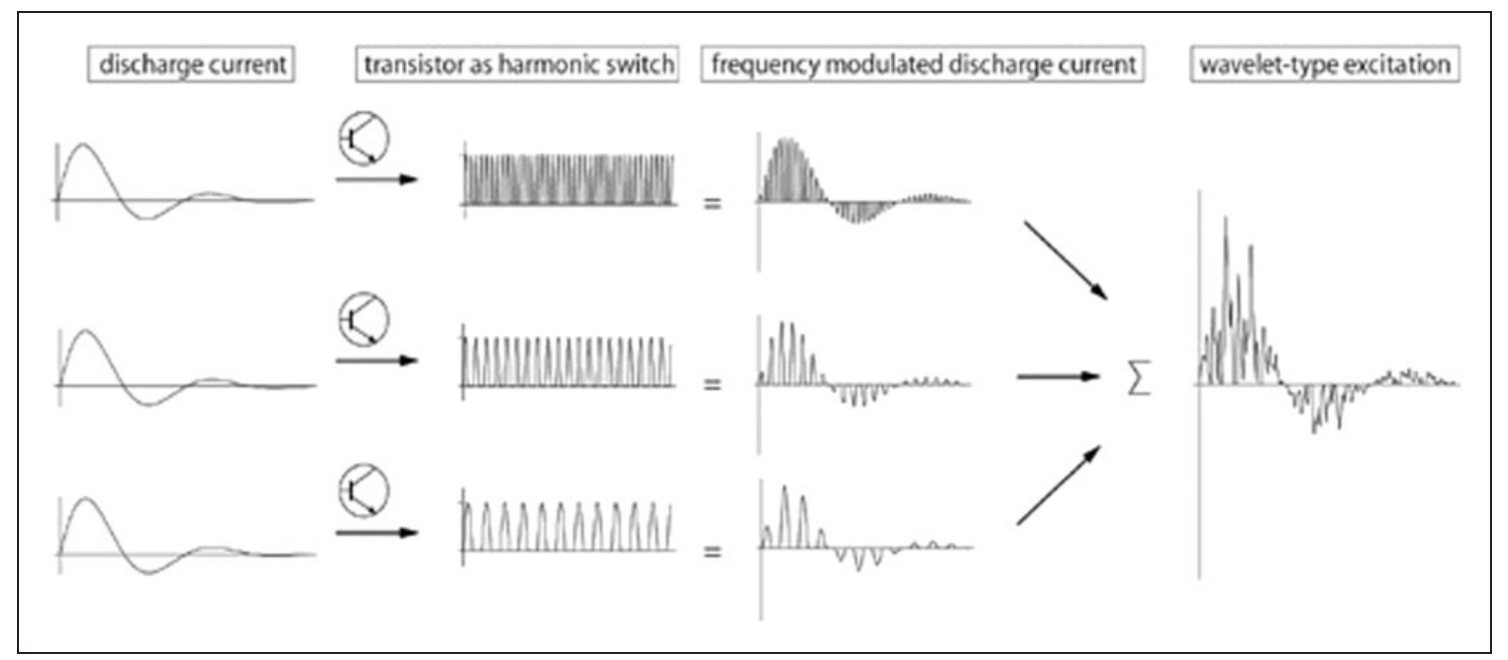

Figure 14. Outlook: producing high-power wavelet-type excitation by frequency modulation of discharge currents.

concerning the temperature dependence of the material's strength. However, concerning the system parameters considered in the present investigation, it finally turned out that the maximum local temperature change is approximately $30 \mathrm{~K}$ and can be neglected in terms of the temperature dependence of material parameters. After calculating the resistance and inductance it turned out that these parameters can be assumed to be constant due to small displacement of the carrying plate relative to the gap between coil and plate which validate the assumption of constant gap between coil and carrying plate and consequently lineary behaving discharge circuit. The displacement of the carrying plate is, just at the discharge time, very small relative to the gap between the coil and the plate.

\section{Results}

A parameter study of five different setups has been done by varying the discharging current's parameters from equation (3) according to Table 2. In setups 1, 2 and 3 different capacitor charges $E_{c}$ and, hence, different charging voltages $U_{0}$ are investigated based on one unaltered circuit with values $L_{a}, C, R_{a}$ according to Figure 6. Setups 4 and 5 additionally adress higher capacitor banks and, therefore, different circuit characteristics. For each setup the SRS at point $\mathbf{P}$ from Figure 8 is calculated in order to study the influence of parameter variation.

Figure 9 shows a comparison of the analytically and experimentally determined currents $I_{1}$ according to equation (3) in Figure 5. It can be distinguished between setups 1, 2, 3 where, due to the constant circuit characteristics, only the current's amplitude increases with increasing charging voltage $U_{0}$ and setups 4, 5 additionally showing different resonance frequencies $\omega$. These tendencies can be recognized in Figure 10 illustrating the resultant of the repulsive Lorentz force simulated based on the FEmodel. 
In Figure 11, the SRS graphs computed with respect to the transversal acceleration data are shown in terms of varying parameters from different setups. Basically, a shift of the SRS graph to higher amplitudes can be observed. Only a negligible effect on the SRS shape by changing the circuit characteristics is resulting from the parameter changes, at least in the range of the five setups.

Additionally, experiments on a high voltage discharge machine for high speed forming have been performed incorporating a quadratic $1 \mathrm{~m}^{2}$ aluminum carrying plate at thickness $h=0.03 \mathrm{~m}$, see Lacher (2011c). The coil was located at one corner beneath the plate, whereas the acceleration data during the discharge process have been measured at the center of the edge opposite to the excitation, as seen in Figure 12.

The experimental SRS results obtained at the measuring point (z-direction) are shown in Figure 13 where special attention has to be turned on the extreme levels of maximum acceleration reaching up to $10,000 \mathrm{~g}$ while, due to the distributional characteristics of the volume forces, no evidence of damage or indentation could be observed subsequently to the experiments conducted.

Considering conventional pyroshock testing techniques, comparable levels are only achieved by the use of explosives which, however, show a wide range of different disadvantages such as poor controllability and repeatability as well as high damage potential.

At this point the authors would like to point out the fact that a comparison of SRS developments between the analytical and experimental results in this paper has not been in the focus of the investigation. Since the SRS projects the dynamic response of the structure to a sudden excitation and is highly affected by the vibration modes of the structure a rotational symmetric domain has been selected for the analytical study, also for the purpose of reducing CPU time. On the other hand, a quadratic plate of the same dimension as the analytical domain diameter has been chosen for handling reasons in order to pursue the experimental part. Therefore the SRS resulting from both analytical and experimental works are not from the same basis and are not really conform to comparison. However, the authors' intention of pointing out the controllability and also the predictability of both, the analytical and experimental SRS tendencies has been successfully presented in this study.

\section{Conclusions and outlook}

The present paper introduces a possible contactless excitation method for pyroshock simulation showing the potential of replacing existing techniques due to a combination of their advantages. The method is based on the discharging process of a simple $R L C$ resonator circuit, the coil of which is approached to a carrying aluminum plate. During the discharging process, an eddy current induces a transient locally distributed repulsive Lorentz body force leading to remarkable acceleration levels within a large bandwidth. The authors present a sophisticated multifield model including the exciting circuit as well as the coupling beween the coil, and the plate by adressing magnetic, thermal and structural coupling. Also, experiments with a high voltage discharge machine and a common carrying structure have been performed leading to very promising results in terms of the potential for practical pyroshock test applications. Regarding the repeatability and controllability as well as the broadband acceleration levels and the nearly vanishing structural damage of the testing equipment, the present mechanism's performance exceeds by far all existing test facilities.

Considering the possibilities of influencing the SRS graph in order to meet test specifications the presented mechanism offers several comfortable approaches which, as an outlook, are proposed by the authors as follows:

1. Wavelet-type excitation by frequency modulation of discharge currents

As seen in Figure 14, the idea consists of charging a number of $R L C$ circuits with identical characteristics. The discharge current of these circuits is then frequency modulated by harmonically switching the current with the help of power transistors.

The switching frequency can be tuned and, hence, serve as system parameter in order to influence the acceleration signal at the specimen's location. An amplifying effect of influencing the SRS can be procured by adjusting the switching frequencies to the resonance frequencies of the stucture carrying the specimen. Compared to common wavelet excitations by piezoelectric actuators (see e.g. Bernaudin et al. (2008) and Roggan (2012)) which are (due to limited deflection capabilities) usually limited by low transferable signal bandwidth and/or power, the proposed mechanism has a significant advantage: the high-power discharge current serves as a carrier of the low-power frequency excitation signal. The large acceleration amplitude coming from the high-power discharging capacitor is frequency modulated by a comparably low-power signal from the power switches. This allows for both in parallel: high power but controlled signal which is a novel combination compared to existing pyroshock test excitation methods. The method which has been 
successfully performed in Roggan (2012) with the help of piezo-actuators can be applied to the method presented.The desired SRS from the test specifications is used to serve as a reference signal for a closed loop control circuit which would directly lead to a preset SRS at a specific point on the specimen without the need of numerical simulation

2. Excitation by multiple coils

An alternative way of influencing the SRS can be found in engaging a number of different coils, each of them having its proper characteristics and, hence, separate discharge currents acting on the carrying plate in terms of a combined and easily controllable Lorentz body force. Both proposed mechanisms show a promising way of influencing the characteristics of SRS in a purely electrical and controllable manner. Finally, an application to near field pyroshock tests is imaginable which could replace the shock excitation by explosives.

\section{Conflict of interest}

The authors declare no conflict of interest.

\section{Funding}

This research received no specific grant from any funding agency in the public, commercial or not-for-profit sectors.

\section{References}

Bäger A (2009) Pyroschockteststand - Erfahrungen und neue Erkenntnisse (Pyroshock test setup - experiences and new expertise). Der Versuchs- und Forschungsingenieur 42.2009: 24-30.

Barboni R, Galluccio G, Sciandra E, et. al. (2003) On the experimental validation of FEM for pyro-shock propagation in space structures. In: Proceedings of the AIDAA XVII Congress, Rome, Italy, 15-19 September, 2003.

Bernaudin JB, et al. (2008) Shock Handbook. ESA Workshop.

Caresta M, Langley RS and Woodhouse J (2014) Transient response of structures with uncertain properties to nonlinear shock loading. Journal of Sound and Vibration 332: 5821-5836.

Davie N and Bateman V (1995) Pyroshock Testing. In: Harris C (ed.) Shock and Vibration Handbook. New York: McGraw-Hill.

Dwyer TJ and Moul D (1988) Pyro Shock Simulation: Experience with the MIPS Simulator. In: Proceedings of the 15th Space Simulation Conference (ed J Stecher), Williamsburg, USA, 31 October-3 November 1988, pp. 125-138. Greenbelt: NASA Goddard Space Flight Center.

Filippi E, Attouoman H and Conti C (1999) Pyroshock Simulation Using the Alcatel Etca Test Facility. In: Proceedings of the first European Conference on
Launcher Technology, Toulouse, France, 14-16 December, 1999.

Henderson G and Piersol A (2003) Evaluating Vibration Environments Using Shock Response Spectrum. Sound and Vibration Magazine 4/2003: 18-20.

Houshmannd B (2010) Stöße und Wellenausbreitung durch piezoelektrische Aktoren (Impact and wave propagation through piezoelectric actuators). Student Research Project, Technische Universität Berlin.

Jang JK and Lee JR (2014) Nondestructive prediction of point source pyroshock response spectra based on experimental conditioning of laser-induced shocks. Optics \& Laser Technology 61: 24-33.

Kiryenko S, Piret G and Kasper J (2005) Shock Bench Presentation. In: Proceedings of the European Conference on Spacecraft Structures, Materials and Mechanical Testing, ESA SP-581, Noordwijk, The Netherlands, 10-12 May, 2005.

Lacher A, Jüngel N, Renning M, et. al. (2011) Response of finite continuous structures with discrete masses to impact and its application to pyroshock simulation. In: Proceedings of the 4th ICMEM, Suzhou, China, 10-12 August, 2011.

Lacher A (2011) Zur analytischen Beschreibung der Stoßantwort einfacher kontinuierlicher Strukturen mit Anwendung auf Pyroschocksimulationen (On the analytical description of the shock response of simple continuous structures with application to pyroshock simulation). $\mathrm{PhD}$ Thesis, Technische Universität Berlin.

Lacher A, Jüngel N, von Wagner U, et al. (2001) Computational simulation of far-field pyroshocks including experiments and optimization. In: Proceedings of the 3rd CEAS Air and Space Conference, Venice, Italy, 17-21 October 2011, pp. 1298-1307.

Lacher A, Jüngel N, von Wagner U, et al. (2012a) Analytical calculation of in-plane response of plates with concentrated masses to impact and application. Journal of Sound and Vibration 331: 3358-3370.

Lacher A, Jüngel N, von Wagner U, et al. (2012b) Analytical prediction and optimazation of far-field pyroshock test procedures. In: Proceedings of the 12th European Conference on Spacecraft Structures, Materials and Environmental Testing, Noordwijk, The Netherlands, 2023 March 2012.

Lalanne C (2005) Mechanical Shock. In: de Silva C (ed.) Vibration and Shock Handbook. Boca Raton: Taylor \& Francis Group12-1-12-61.

Lee JR, Chia CC and Kong CW (2012) Review of pyroshock wave measurement and simulation for space systems. Journal of the International Measurement Confederation 45: 631-642.

Roggan R (2012) Untersuchungen zur Anwendung eines Piezoaktors zur Erzeugung komplexer Schocksignale (Investigations on the application of a piezoactuator for generating complex shock signals). Diploma Thesis, Technische Universität Berlin.

Schweickert G (1997) The Dornier Shocktable - A new Facility for Shocktesting of Components. In: Proceedings of the Third International Symposium on Environmental Testing for Space Programs, ESA 
SP-408, Noordwijk, The Netherlands, 25-27 June 1997, pp. $145-150$.

Smith JL (1986) Effects of Variables Upon Pyrotechnically Induced Shock Spectra. NASA Technical Paper: 2603.

Stewart LK, Durant B, Wolfson J, et al. (2014) Experimentally generated high-g shock loads using Hydraulic Blast Simulator. International Journal of Impact Engineering 69: 86-94.

Uhlmann E and Ziefle A (2010) Modeling Pulse Magnetic Welding Processes an Empirical Approach. In: Proceedings of the 4th International Conference on High Speed Forming, Columbus, USA, 9-10 March 2010.

Unger J, Stiemer M, Svendsen B, et al. (2006) Multifield modeling of electromagnetic metal forming processes. Journal of Materials Processing Technology 177: $270-273$

Winkler R (1973) Hochgeschwindigkeitsbearbeitung. Grundlagen und technische Anwendung elektrisch erzeugter Schockwellen und Impulsmagnetfelder (High-speedforming: fundamentals and technical application of eletrically generated shockwaves and impulse magnetic fields). Berlin: Verlag Technik.

$\mathrm{Xu} \mathrm{W}$, Fang $\mathrm{H}$ and $\mathrm{Xu}$ W (2008) Analysis of the variation regularity of the parameters of the discharge circuit with the distance between workpiece and inductor for electromagnetic forming processes. Journal of Materials Processing Technology 203: 216-220. 